\title{
Blood Pressure and Heart Rate Measurements Using Photoplethysmography with Modified LRCN
}

\author{
Chih-Ta Yen ${ }^{1, *}$ and Cheng-Hong Liao ${ }^{2}$ \\ ${ }^{1}$ Department of Electrical Engineering, National Taiwan Ocean University, Keelung City, 202301, Taiwan \\ ${ }^{2}$ Department of Electrical Engineering, National Formosa University, Yunlin County, 632, Taiwan \\ *Corresponding Author: Chih-Ta Yen. Email: chihtayen@gmail.com \\ Received: 15 August 2021; Accepted: 18 September 2021
}

\begin{abstract}
In this study, single-channel photoplethysmography (PPG) signals were used to estimate the heart rate (HR), diastolic blood pressure (DBP), and systolic blood pressure (SBP). A deep learning model was proposed using a long-term recurrent convolutional network (LRCN) modified from a deep learning algorithm, the convolutional neural network model of the modified inception deep learning module, and a long short-term memory network (LSTM) to improve the model's accuracy of BP and HR measurements. The PPG data of 1,551 patients were obtained from the University of California Irvine Machine Learning Repository. How to design a filter of PPG signals and how to choose the loss functions for deep learning model were also discussed in the study. Finally, the stability of the proposed model was tested using a 10-fold cross-validation, with an MAE \pm SD of $2.942 \pm 5.076$ $\mathrm{mmHg}$ for SBP, $1.747 \pm 3.042 \mathrm{mmHg}$ for DBP, and $1.137 \pm 2.463 \mathrm{bpm}$ for the HR. Compared with its existing counterparts, the model entailed less computational load and was more accurate in estimating SBP, DBP, and HR. These results established the validity of the model.
\end{abstract}

Keywords: Photoplethysmography (PPG) signal; deep learning; blood pressure; systolic blood pressure (SBP); diastolic blood pressure (DBP); heart rate (HR)

\section{Introduction}

Cardiovascular diseases (CVDs) are one of the world's leading causes of death. Common CVDs include heart disease, cerebrovascular disease, hypertension, nephritis, nephrotic syndrome, and nephropathy. One effective way to prevent CVDs is measuring blood pressure (BP) regularly. $\mathrm{BP}$ is typically measured using a mercury sphygmomanometer, electronic sphygmomanometer and electrocardiography [1]. Mercury sphygmomanometers are highly accurate but not straightforward to use [2]. Electronic sphygmomanometers and electrocardiography are more convenient but have numerous usage limits. Both mercury and electronic sphygmomanometers are discontinuous, cuffbased methods for measuring BP. Electrocardiography requires a number of electrodes to be attached to the body, which is an inconvenience when it comes to measuring BP continuously [3-5].

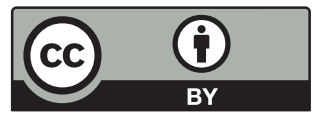

This work is licensed under a Creative Commons Attribution 4.0 International License, which permits unrestricted use, distribution, and reproduction in any medium, provided the original work is properly cited. 
Photoplethysmography (PPG), which absorbs light energy through a photodetector, can be used to monitor a patient's pulse rate. Composed of a light-emitting diode (LED), photodiode, and amplifier, it is a low-cost optical method that can be used to detect changes in the blood volume of the capillary beds of body tissues, because the induced voltage of the photodetector changes along with the perunit-area volume of blood flow in the vessels, which varies as the heart pulses. The detector absorbs the most light when the heart contracts; therefore, the volume of blood entering and exiting tissues is linked with the amplitude of the PPG signal. The PPG waveform consists of direct-current (DC) and alternating-current (AC) components, with the former corresponding to the transmitted or reflected light signal detected in tissues and depending on the tissue structures and the blood volume in veins, and the latter changing with breathing, representing how blood volume varies with breathing between the systolic and diastolic phases of the cardiac cycle, and having its signal superimposed on the DC signal. PPG was first used in clinical settings in 1937, when Hertzman and Spielman proposed its use as a device that utilized lightwaves to measure changes in the volume of blood flowing through the patient's fingers, with the measurement results applied in future research [6].

In recent years, deep learning has been applied to solve medical problems, particularly in terms of PPG-based BP measurement, which eliminates the need to extract features manually. In particular, a PPG spectrogram can be input into an artificial neural network (ANN), so that the ANN is trained to estimate PPG features and determine BP values accordingly. The researchers used deep recurrent neural networks (RNNs) to learn the long- and short-term features from patients' PPG data [7,8]. The scholars used a Siamese neural network that obtained near-actual BP values taking into account PPG signals [9]. The authors proposed "PP-Net," a deep learning model that estimates systolic pressure, diastolic pressure, and the heart rate and is built around a convolutional neural network (CNN) and a long short-term memory (LSTM) network [10]. All of these researchers have tested their proposed methods on small groups of healthy subjects. Thus, the testing of deep learning methods for BP estimation among different groups of patients with CVD complications is critical and essential for facilitating the clinical application of these methods, particularly when it comes to monitoring the heart condition and the risk of stroke in critically ill patients postoperatively. Several researchers have discussed the relationship between BP and PPG in the context of cuff-less estimation [11-15]. However, many of them have used machine learning algorithms and manually extracted features before BP estimation - $\mathrm{a}$ job that is complicated and entails considerable calculation. Compared with traditional machine learning methods for BP measurement, deep learning models can learn high-dimensional features and are highly capable of modeling complex, nonlinear systems. Moreover, deep learning algorithms eliminate the need to acquire features manually and are thus a primary tool in a number of biomedical applications [16-21].

Loss functions can be estimated using the mean absolute error, which originates from the mean error and represents the square form of the mean square error (MSE) [22]. The researchers presented a theoretical analysis of an upper bound on a generalized loss of MAE for deep neural network (DNN)-based vector-to-vector regression [23]. The authors used the Huber function to perform robust estimation in electrical power engineering [24]. The researchers discussed the effectiveness of the MAE and observed how the error varied when they trained a deep learning model to predict energy load [25]. The scholars studied the distributional loss for regression from the perspective of effective optimization, examining the MAE and MSE [26]. The authors applied MAE and MSE loss functions in a generative adversarial network [27]. The researchers studied the role of the MAE loss function in DNN-based vector-to-vector regression; they showed that optimizing the regression with the MAE loss function achieved less loss than with the MSE equivalent, thus leading to higher accuracy during 
model training [28]. Given the cited works, one can conclude that using a good loss function can effectively improve the performance of a deep-learning neural network.

In the present study, a deep learning framework was developed using a PPG sensor built on a modified long-term recurrent convolutional network (LRCN) that consisted of a CNN and an LSTM network [16]. The proposed CNN-LSTM framework-which leveraged the strengths of both neural networks and the feature extraction capability of the inception module - functioned as a highperformance model. The novelty of this design resides in a deep learning framework that features a customized model and a multi-output capability to estimate the diastolic blood pressure (DBP), systolic blood pressure (SBP), and the heart rate (HR) simultaneously. Such a framework has been successfully tested with the cuff-less blood pressure estimation dataset, which consists of monitoring data for ICU patients with different CVD complications [29]. After using the framework to examine the data derived from 1,551 patients, we obtained a normalized mean absolute error (NMAE) of 0.0121 , normalized root mean square error of 0.0227 , and correlation coefficient of 0.97 . The deep learning model can also be used to determine DBP, SBP, and the HR, which justifies its role in examining physiological information by using the time-series data collected via PPG, and its robustness is confirmed by its ability to perform calculations. All of these suggest that the model can be applied in the comprehensive medical monitoring of patients with diabetes or stroke, elderly patients, and patients who have undergone surgery. In addition, the model's effectiveness in estimating BP and HR was validated through a comparison with the existing estimation methods. The rest of this paper is organized as follows. Section 2 describes the proposed method. Section 3 provides an analysis of the results. Section 4 presents the conclusions.

\section{Method}

Considering the strengths of PPG and the gaps in the existing literature, we propose a highperformance deep learning framework with a multi-output capability to estimate DBP, SBP, and HR. This framework was studied using the cuff-less blood pressure estimation dataset from the University of California Irvine (UCI) Machine Learning Repository. This dataset was derived from the openaccess Medical Information Mark for Intensive Care II (MIMIC-II), which can be obtained from the PhysioNet repository and consists of different categories of medical information relating to ICU patients, which include physiological signals and parameters [30]. The PPG and arterial blood pressure (ABP) records of 12,000 patients were sampled from the UCI Machine Learning Repository at a frequency of $125 \mathrm{~Hz}$. The training and test values for SBP and DBP were acquired using the method proposed in [31]; the method involves extracting the crests and troughs of ABP signals. Moreover, the number of complete waveforms was estimated to determine the HR, because a full HR reading includes the SBP and DBP values. The proposed deep learning framework was used in a PPG sensor to estimate SBP, DBP, and HR simultaneously, thereby ensuring its use in comprehensive medical monitoring.

\subsection{Stage 1: Preprocessing}

Preprocessing was performed according to reference [19]. In particular, PPG data with a recording duration of $<8 \mathrm{~min}$ were removed; data segmentation was then conducted taking an $8 \mathrm{~s}$ window with $75 \%$ overlapping. Next, a band-pass fourth-order Chebyshev II filter was used to process the PPG data at the $0.5-10 \mathrm{~Hz}$ frequency [10]. Next, down-sampling was performed to cut the number of subjects for the $8 \mathrm{~s}$ window from 1,000 to 250 (Fig. 1) so as to reduce the computational load of the deep learning 
model. Finally, the PPG data, as well as their corresponding SBP and DBP values, were treated with min-max normalization and then used in the model.

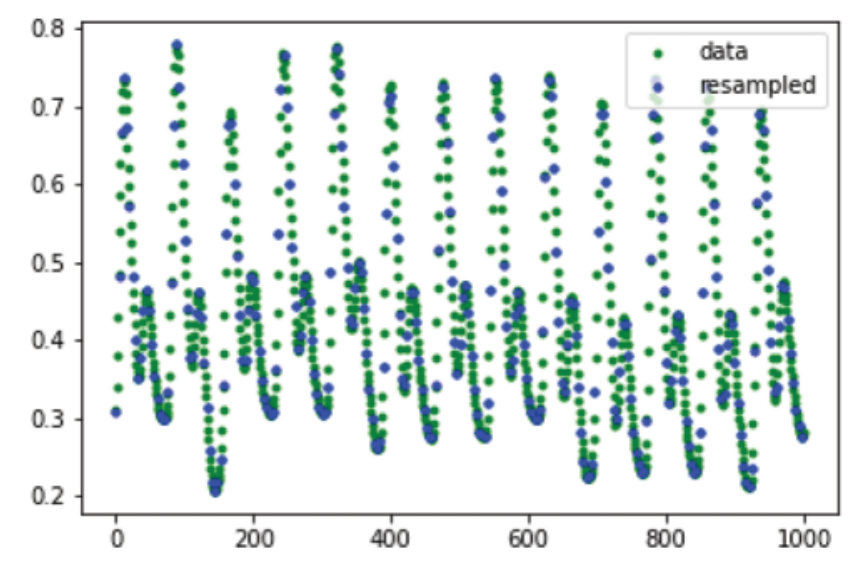

Figure 1: Raw data vs. down-sampled data

\subsection{Stage 2: Deep Learning Model}

Our deep learning framework, which used a CNN and an LSTM network, was defined as a modified LRCN model. Its network structure, designed to achieve multiple outputs, could estimate DBP, SBP, and HR simultaneously when analyzing PPG signals. Fig. 2 describes the topology of the LRCN model, which shows the stacking of the multi-scale CNN, the LSTM network, and a dense layer. Within this topology, a multi-convolution module and a convolution layer served as the feature extractor; the convolution module consisted of two 1-dimensional convolution layers, BatchNormalization layers, and ReLU activation layers. The module output features to a maxpooling layer; the features entered a 1-dimensional convolution layer, BatchNormalization layer, ReLU activation layer, and maxpooling layer sequentially. The output features acquired from the last maxpooling layer then passed through two LSTM layers and were delivered to the dense layer for the estimation of the three physiological parameters.

Fig. 3 depicts the proposed model's architecture, which consisted of a 1-dimensional multiconvolution module, two LSTM layers, and a dense layer. The model was built on a CNN in which two multi-convolution modules - one was composed of ten $9 \times 1$ kernel filters, normalized through batch normalization, and activated through the ReLU function, whereas the other was composed of ten $25 \times 1$ filters, normalized through batch normalization, and activated through the ReLU functionhad their feature vectors concatenated. The modules were maxpooled within the spatial dimensions of $9 \times 1$, taking into account the inception module, whose increased network width enabled it to capture more details and features. The 1-dimensional multi-convolution module was composed of a set of $329 \times 1$ filters, normalized through batch normalization, activated through the ReLU activation function, and maxpooled within the spatial dimensions of $3 \times 1$.

Next, the 64 and 128 memory cells of the two LSTM layers for the hyperbolic tangent function "tanh" were integrated with the CNN model to solve regression problems. A dense layer that contained three output neurons was used with a linear function to perform prediction, each of these neurons predicted DBP, SBP, and HR, respectively. To ease overfitting for the proposed model, feature vectors from the convolution layers were normalized through batch normalization. 
CMC, 2022, vol.71, no.1

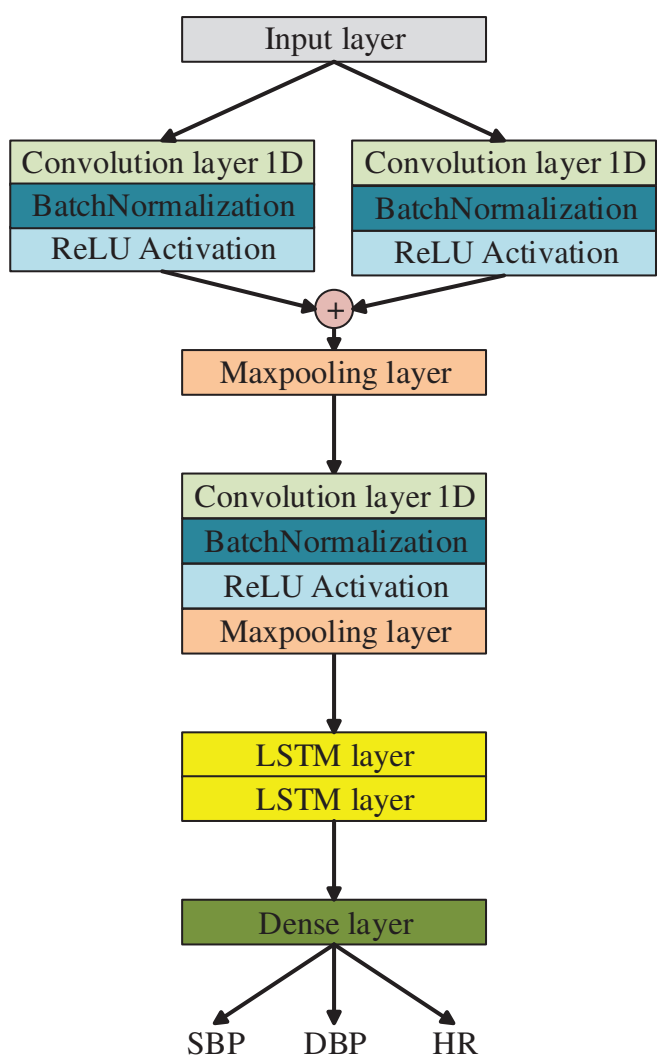

Figure 2: Topology of the proposed multi-scale LRCN model

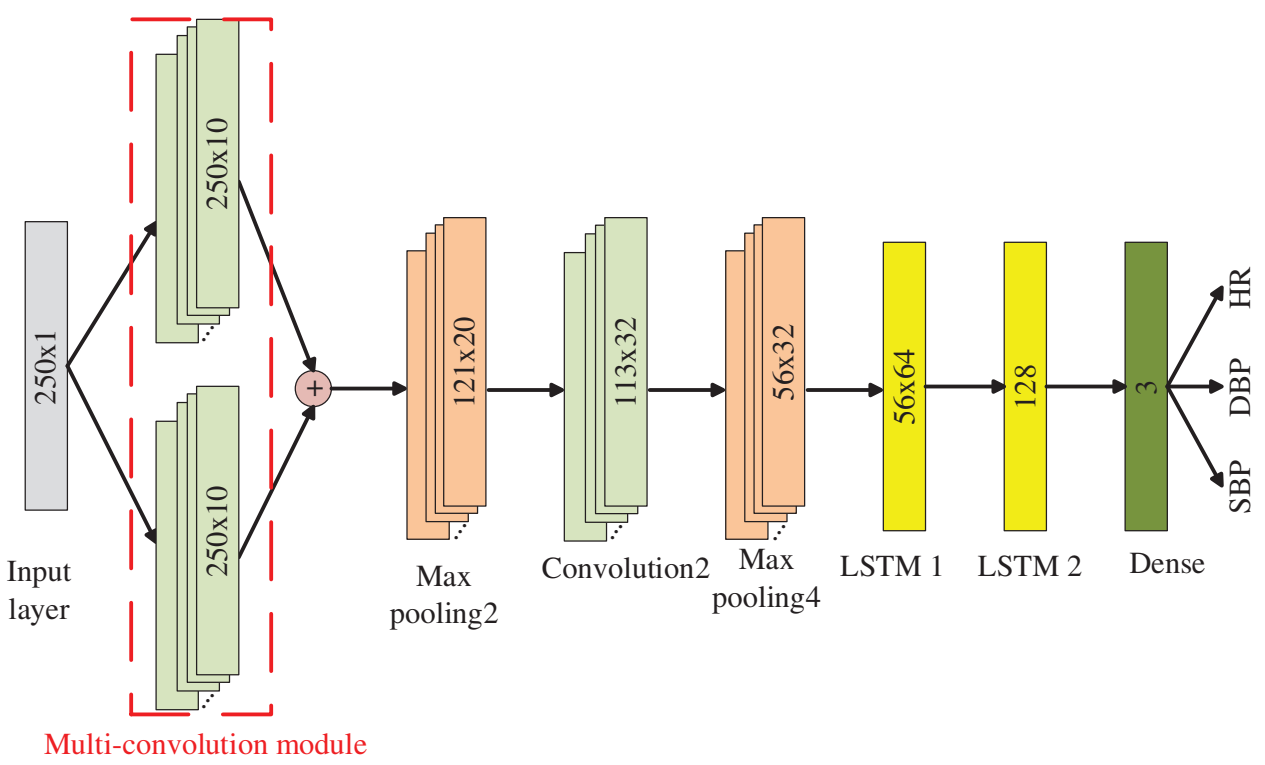

Figure 3: Architecture of the proposed multi-convolution model 


\subsection{Stage 3: Training and Evaluation}

The deep learning model was trained using 50 batches with a size of 100 . During training, the model was optimized using the Adam optimizer. The MSE, MAE and Huber were used as a loss functions to evaluate the proposed deep learning framework's performance. The model underwent $k$-fold cross-validation because this training/testing method can be used to test a model's generalizability [20]. In our experiment, $k$ was set to 10 , so the model's performance was determined on the basis of the average prediction score by using 10 testing datasets.

\section{Analysis of Results}

The deep learning framework used in the experiment was realized with Kera 2.4.3, which uses Tensorflow 2.4.0 as the backend engine. The hardware setup for the experiment consisted of a 6-GB Nvidia GEFORCE RTX 2060 GPU, which trained, validated, and tested the model, and was implemented on a 64-bit Windows operating system with an Intel Intel(R) Core(TM) i7-8700 CPU @ 3.20 GHz and 32-GB RAM.

\subsection{Deep Learning Model's Performance Across Different Loss Functions}

Tab. 1 shows the performance of the proposed deep learning model as estimated by three different loss functions.

A loss function is typically expressed as the MSE or MAE. Thus, the MSE and MAE were included in our analysis of the model's performance. The performance was determined through both loss functions, and the results were compared with those obtained using the Huber loss function. As shown in Tab. 1, the model exhibited the highest performance with the MAE as a loss function.

Table 1: Performance of the proposed deep learning model estimated by different loss functions (with the units of the error measures for both BP values and HR expressed, respectively, in $\mathrm{mmHg}$ and bpm)

\begin{tabular}{llll}
\hline Parameter & \multicolumn{2}{l}{ LOSS function } & \\
\cline { 2 - 4 } & MSE & MAE & Huber \\
\hline SBP MAE & 3.139 & 2.942 & 3.34 \\
DBP MAE & 1.927 & 1.747 & 2.005 \\
HR MAE & 1.783 & 1.137 & 1.814 \\
\hline
\end{tabular}

\subsection{Role of Chebyshev II Filter in the Preprocessing of Input Data}

As mentioned earlier, PPG signals were filtered through a Chebyshev II filter to attenuate the noise of the measured signals and improve the proposed model's estimation accuracy. Therefore, a comparison was made in the model's estimation performance using PPG data preprocessed by the filter $v s$. the PPG data not preprocessed by the filter.

The difference of overall estimates of SBP, DBP and HR of NMAE with/without PPG data preprocessed by the Chebyshev II filter was 0.0019 as shown in Tab. 2. These results suggested that the expected PPG waveforms derived from the filter directly affected the proposed deep learning 
model's estimation accuracy. Thus, preprocessing PPG signals through the designed Chebyshev II filter effectively improved the model's accuracy.

Table 2: Model performance with data preprocessed by a Chebyshev II filter $v s$. not preprocessed by the filter (the error measures for both BP values and the HR are expressed, respectively, in $\mathrm{mmHg}$ and bpm)

\begin{tabular}{lll}
\hline Filter & \multicolumn{2}{l}{ Chebyshev II filter } \\
\cline { 2 - 3 } & Used & Not used \\
\hline SBP NMAE & 0.0163 & 0.0174 \\
DBP NMAE & 0.0134 & 0.0141 \\
HR NMAE & 0.0065 & 0.0066 \\
\hline
\end{tabular}

\subsection{Performance Evaluation}

One objective of this experiment was to build a deep learning model that, even with fewer layers, could perform satisfactorily to enable real-time monitoring on a mobile platform. Thus, the model was developed by modifying an LRCN model in ways that replaced a CNN layer with an inception module and adjusted the model to estimate DBP, SBP, and HR simultaneously, which are otherwise evaluated through separate models. Moreover, downsampling was performed to filter the PPG signals input into the model, thereby reducing the complexity of data processing and thus the calculation load of the model. Our model was modified according to reference [10], with the number of data points reduced from 1,000 to 250 after downsampling.

Tab. 3 presents the performance of the proposed model evaluated through a 10 -fold crossvalidation, with the overall NMAE averaging 0.0121 and NRMSE 0.0228 among SBP, DBP and HR. Fig. 4 presents a comparison of the true and predicted values for SBP, DBP and HR obtained through 500 tests, with the true and predicted values colored in blue and orange, respectively. Fig. 5 depicts the normal distribution of errors for SBP, DBP and HR.

Table 3: Performance of the proposed deep learning model (with the units of the error measures for both $\mathrm{BP}$ values and the $\mathrm{HR}$ expressed, respectively, in $\mathrm{mmHg}$ and $\mathrm{bpm}$ )

\begin{tabular}{lll}
\hline Parameter & \multicolumn{2}{l}{ Performance } \\
\cline { 2 - 3 } & NMAE & NRMSE \\
\hline SBP & 0.01634 & 0.7532 \\
DBP & 0.01345 & 0.5561 \\
HR & 0.00659 & 0.5387 \\
\hline
\end{tabular}

\subsection{Proposed Method vs. Existing Methods}

1. BP: The proposed method was compared with the existing methods in terms of feasibility. While the existing methods involve feature selection and extraction, our model-which yielded 
an MAE \pm SD of $2.345 \pm 4.059 \mathrm{mmHg}$ by estimating $\mathrm{BP}$ with data from 1,551 subjects-showed better performance (Tab. 4). This result confirmed our model's feasibility in clinical settings.

2. HR: Tab. 5 presents a comparison of the performance of HR estimation between the proposed method and the existing methods. Compared with most of the existing models, our model used more data to determine the HR to achieve greater accuracy and generalizability. In addition, while numerous researchers have focused specifically on the HR (Tab. 5), we examined DBP, SBP, and HR, although this led to an increased training load in our model. However, our model performed almost as good as some existing models, and better than the others, with an $\mathrm{MAE} \pm \mathrm{SD}$ of $1.137 \pm 2.463 \mathrm{bpm}$.

We estimated DBP, SBP, and HR simultaneously, without having to train our model for each of the parameters separately. Therefore, the model might require less computational load in the context of real-time system analysis.

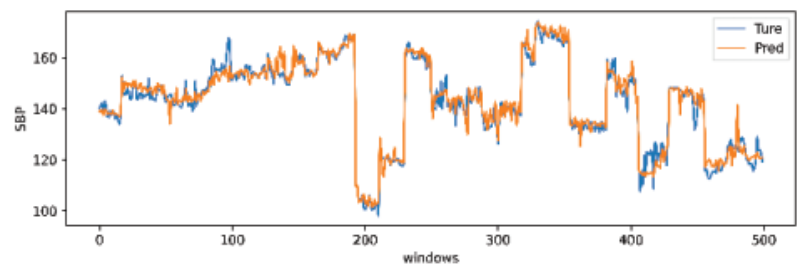

(a)

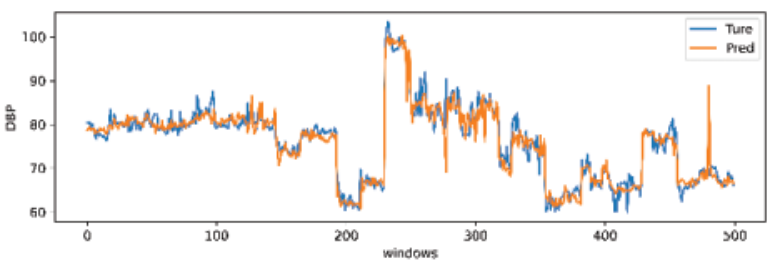

(b)

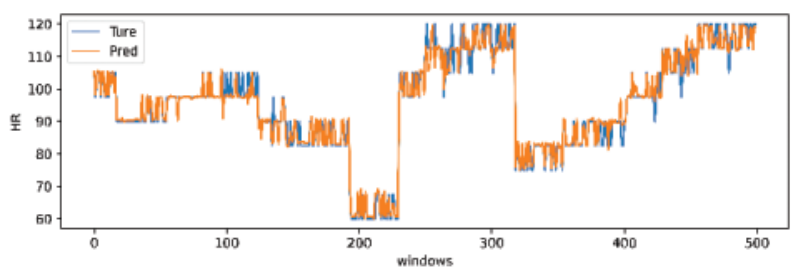

(c)

Figure 4: True vs. predicted values obtained through 500 tests: (a) SBP, (b) DBP, and (c) HR

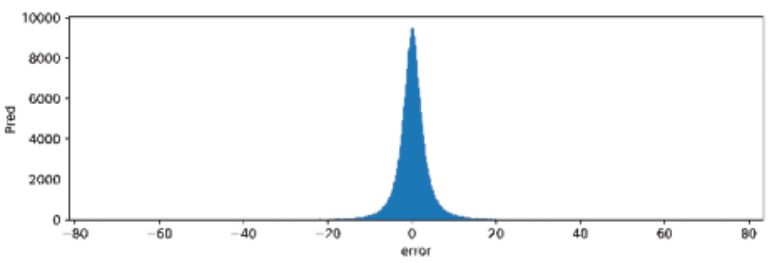

(a)

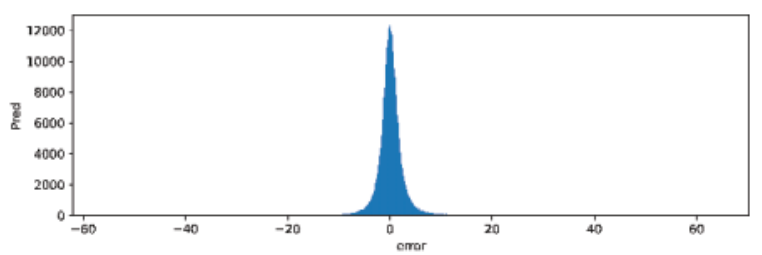

(b)

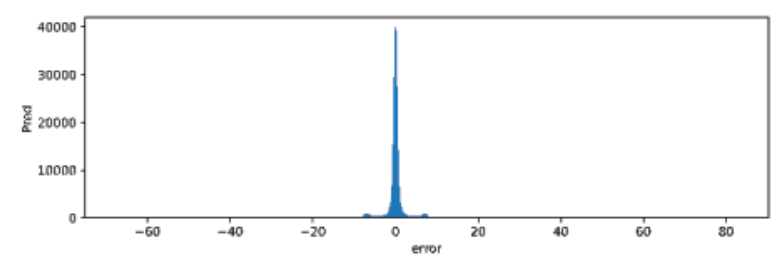

(c)

Figure 5: Normal distributions of errors for (a) SBP, (b) DBP, and (c) HR 
Table 4: BP estimation performance of proposed method $v$ s. existing methods (with the error measures for SBP and DBP expressed in $\mathrm{mmHg}$ )

\begin{tabular}{|c|c|c|c|c|c|}
\hline \multirow[t]{2}{*}{ Work } & \multirow[t]{2}{*}{ Subjects } & \multirow[t]{2}{*}{ Method } & \multirow{2}{*}{$\begin{array}{l}\text { Validation } \\
\text { method }\end{array}$} & \multicolumn{2}{|c|}{ Performance $(\mathrm{MAE} \pm \mathrm{SD})(\mathrm{mmHg})$} \\
\hline & & & & SBP & DBP \\
\hline $\begin{array}{l}\text { EMBC'16 } \\
\text { Gaurav } \\
\text { et al. [13] }\end{array}$ & $\begin{array}{l}3,000 \\
\text { subjects }\end{array}$ & $\begin{array}{l}\text { Feature extraction } \\
(46 \text { features) and } \\
\text { artificial neural } \\
\text { network (ANN) }\end{array}$ & $\begin{array}{l}\text { Conventional } \\
\text { method }\end{array}$ & $4.47 \pm 6.85$ & $3.21 \pm 4.72$ \\
\hline $\begin{array}{l}\text { TBME'16 } \\
\text { Kachuee } \\
\text { et al. [14] }\end{array}$ & $\begin{array}{l}1,000 \\
\text { subjects }\end{array}$ & $\begin{array}{l}\text { DWT, PCA, } \\
\text { whole based, and } \\
\text { physiological } \\
\text { feature extraction } \\
\text { and conventional } \\
\text { regression } \\
\text { algorithms }\end{array}$ & $\begin{array}{l}\text { 10-fold } \\
\text { validation }\end{array}$ & $11.17 \pm 10.09$ & $5.35 \pm 6.14$ \\
\hline $\begin{array}{l}\text { BSPC'18 } \\
\text { Mousavi } \\
\text { et al. [15] }\end{array}$ & $\begin{array}{l}441 \\
\text { subjects }\end{array}$ & $\begin{array}{l}\text { FFT, FFT1, } \\
\text { Feature } \\
\text { extraction, PCA, } \\
\text { and conventional } \\
\text { regression } \\
\text { algorithms }\end{array}$ & $\begin{array}{l}\text { 10-fold } \\
\text { validation }\end{array}$ & $3.97 \pm 8.901$ & $2.43 \pm 4.173$ \\
\hline $\begin{array}{l}\text { ICASSP } \\
\text { Schlesinger } \\
\text { et al. [9] }\end{array}$ & $\begin{array}{l}304 \\
\text { subjects }\end{array}$ & $\begin{array}{l}\text { Siamese learning } \\
\text { Algorithm }\end{array}$ & $\begin{array}{l}\text { Conventional } \\
\text { method }\end{array}$ & $5.95 \pm 6.69$ & $3.41 \pm 3.97$ \\
\hline $\begin{array}{l}\text { IEEE } \\
\text { Sensors } \\
\text { Journal } \\
\text { Panwar } \\
\text { et al. [10] }\end{array}$ & $\begin{array}{l}1,557 \\
\text { subjects }\end{array}$ & $\begin{array}{l}\text { LRCN deep } \\
\text { learning } \\
\text { Algorithm }\end{array}$ & $\begin{array}{l}10 \text {-fold } \\
\text { validation }\end{array}$ & $3.97 \pm 5.41$ & $2.30 \pm 5.65$ \\
\hline $\begin{array}{l}\text { EMBC'20 } \\
\text { Kyriacou } \\
\text { et al. [32] }\end{array}$ & & $\begin{array}{l}\text { Feature extraction } \\
\text { ( } 7 \text { features) } \\
\text { and recurrent } \\
\text { network models }\end{array}$ & & $\begin{array}{l}\text { Neural } \\
\text { network (NN): } \\
4.23 \pm 4.78 \\
\text { Long } \\
\text { short-term } \\
\text { memory } \\
\text { (LSTM): } \\
3.23 \pm 4.74 \\
\text { Gated } \\
\text { recurrent units } \\
\text { (GRUs): } \\
3.25 \pm 4.76\end{array}$ & $\begin{array}{l}\text { Neural } \\
\text { network (NN): } \\
2.37 \pm 2.26 \\
\text { Long } \\
\text { short-term } \\
\text { memory } \\
\text { (LSTM): } \\
1.59 \pm 1.96 \\
\text { Gated } \\
\text { recurrent units } \\
\text { (GRUs): } \\
1.43 \pm 1.77\end{array}$ \\
\hline This work & $\begin{array}{l}1,551 \\
\text { subjects }\end{array}$ & & $\begin{array}{l}10 \text {-fold } \\
\text { validation }\end{array}$ & $2.942 \pm 5.065$ & $1.747 \pm 3.042$ \\
\hline
\end{tabular}


Table 5: HR estimation performance of proposed method vs. existing methods

\begin{tabular}{|c|c|c|c|c|}
\hline \multirow[t]{2}{*}{ Work } & \multirow[t]{2}{*}{ Subjects } & \multirow[t]{2}{*}{ Method } & \multirow[t]{2}{*}{$\begin{array}{l}\text { Validation } \\
\text { method }\end{array}$} & \multirow{2}{*}{$\begin{array}{l}\text { Performance } \\
(\mathrm{MAE} \pm \mathrm{SD})\end{array}$} \\
\hline & & & & \\
\hline $\begin{array}{l}\text { TBME'15 } \\
\text { Zhang } \\
\text { et al. [33] }\end{array}$ & 23 subjects & $\begin{array}{l}\text { Signal processing } \\
\text { method (signal } \\
\text { decomposition, temporal } \\
\text { difference, sparse signal } \\
\text { reconstruction, spectral } \\
\text { peak tracking) }\end{array}$ & & $\begin{array}{l}2.34 \pm 2.47 \\
1-12 \text { subjects } \\
3.19 \pm 3.61 \\
13-23 \text { subjects } \\
2.77 \pm 3.0423 \\
\text { subjects }\end{array}$ \\
\hline $\begin{array}{l}\text { TBME'15 } \\
\text { Zhang } \\
\text { [34] }\end{array}$ & 23 subjects & $\begin{array}{l}\text { Signal processing } \\
\text { method (joint sparse } \\
\text { spectral reconstruction, } \\
\text { spectral subtraction, } \\
\text { peak tracking) }\end{array}$ & & $\begin{array}{l}1.28 \pm 2.61 \\
1-12 \text { subjects } \\
3.05 \pm 3.35 \\
13-23 \text { subjects } \\
2.17 \pm 2.98\end{array}$ \\
\hline $\begin{array}{l}\text { TBME' } 16 \\
\text { Khan } \\
\text { et al. [35] }\end{array}$ & 12 subjects & $\begin{array}{l}\text { Signal processing } \\
\text { method (ensemble } \\
\text { empirical mode } \\
\text { decomposition, adaptive } \\
\text { filtering, decision } \\
\text { making processing) }\end{array}$ & & $\begin{array}{l}1.02 \pm 1.79 \\
1-12 \text { subjects }\end{array}$ \\
\hline $\begin{array}{l}\text { TBME'17 } \\
\text { Tempko } \\
{[36]}\end{array}$ & 23 subjects & $\begin{array}{l}\text { Signal processing } \\
\text { method-WFPV algorithm } \\
\text { (pre-process, de-nosing } \\
\text { phase decoder, post } \\
\text { processing) }\end{array}$ & & $\begin{array}{l}1.02 \pm 1.25 \\
1-12 \text { subjects } \\
2.95 \pm 3.71 \\
13-23 \text { subjects } \\
1.99 \pm 2.0048 \\
\text { subjects }\end{array}$ \\
\hline $\begin{array}{l}\text { Sensors } \\
\text { Letters } \\
\text { Zhu } \\
\text { et al. [37] }\end{array}$ & 12 subjects & $\begin{array}{l}\text { Neural network, linear } \\
\text { regression, post } \\
\text { processing }\end{array}$ & & $\begin{array}{l}1.03 \pm 1.8212 \\
\text { subjects }\end{array}$ \\
\hline $\begin{array}{l}\text { BSPC } * 19 \\
\text { KR } \\
\text { et al. [38] }\end{array}$ & 12 subjects & $\begin{array}{l}\text { Signal processing } \\
\text { method (cascaded three } \\
\text {-stage adaptive filters, } \\
\text { FFT) }\end{array}$ & & $\begin{array}{l}0.92 \pm 1.17 \\
1-12 \text { subjects }\end{array}$ \\
\hline $\begin{array}{l}\text { TBioCAS'19 } \\
\text { Biswas } \\
\text { et al. [39] }\end{array}$ & 25 subjects & $\begin{array}{l}\text { CNN and LSTM deep learning } \\
\text { Algorithm }\end{array}$ & $\begin{array}{l}5 \text {-fold } \\
\text { validation }\end{array}$ & $\begin{array}{l}1.99 \pm 4.6412 \\
\text { subjects } \\
0.86 \pm 1.86 \\
13-23 \text { subjects } \\
1.47 \pm 3.37 \\
25 \text { subjects }\end{array}$ \\
\hline $\begin{array}{l}\text { IEEE } \\
\text { Sensors } \\
\text { Journal } \\
\text { Panwar } \\
\text { et al. [10] }\end{array}$ & $\begin{array}{l}12,000 \\
\text { subjects }\end{array}$ & $\begin{array}{l}\text { LRCN deep learning } \\
\text { algorithm }\end{array}$ & $\begin{array}{l}\text { 10-fold } \\
\text { validation }\end{array}$ & $\begin{array}{l}2.32 \pm 0.095 \\
12,000 \\
\text { subjects }\end{array}$ \\
\hline This work & 1,551 subjects & $\begin{array}{l}\text { LRCN deep learning } \\
\text { algorithm }\end{array}$ & $\begin{array}{l}10 \text {-fold } \\
\text { validation }\end{array}$ & $\begin{array}{l}1.137 \pm 2.463 \\
1,551 \text { subjects }\end{array}$ \\
\hline
\end{tabular}




\subsection{Verification of Proposed Model by British Hypertension Society (BHS) Standards}

The BHS standards were used as the yardstick to determine the validity of our model's BP estimation results. By these standards, the model's BP estimation accuracy was examined in terms of the cumulative error percentage across three grades (Tab. 6). The model's BP estimation results - based on data from 1,551 subjects - were verified according to the standards, satisfying Grade A for the SBP and DBP measurements (Tab. 7). The correlation coefficients of DBP (with data points colored in red) and SBP (with data points colored in blue) are plotted in Fig. 6.

Table 6: BHS grading scale for BP estimation

\begin{tabular}{llll}
\hline Grade & \multicolumn{3}{l}{ Cumulative error percentage } \\
\cline { 2 - 4 } & $\leq 5$ & $\leq 10$ & $\leq 15$ \\
\hline A & $60 \%$ & $85 \%$ & $95 \%$ \\
B & $50 \%$ & $75 \%$ & $90 \%$ \\
C & $40 \%$ & $65 \%$ & $85 \%$ \\
\hline
\end{tabular}

Table 7: Proposed model's BP estimation performance under BHD standards

\begin{tabular}{lllll}
\hline Method & $\begin{array}{l}\text { Physiological } \\
\text { parameter }\end{array}$ & $\leq 5(\mathrm{mmHg})$ & $\leq 10(\mathrm{mmHg})$ & $\leq 15(\mathrm{mmHg})$ \\
\hline This & SBP & $92 \%$ & $97 \%$ & $98 \%$ \\
work & DBP & $96 \%$ & $99 \%$ & $99 \%$ \\
\hline
\end{tabular}

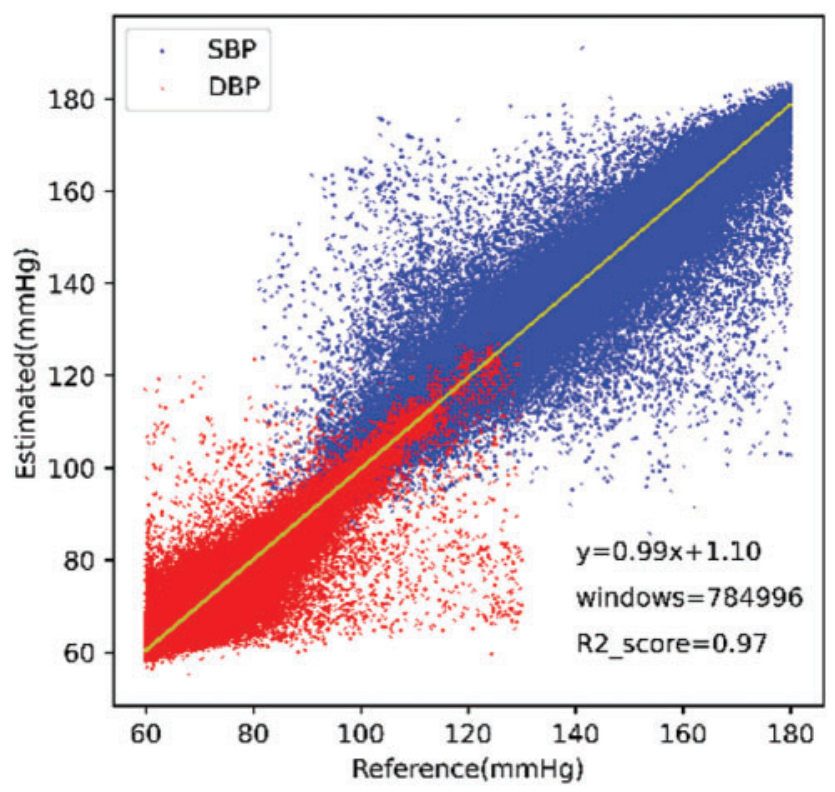

Figure 6: Scatter plot of SBP (blue) and DBP (red) 


\section{Conclusion}

Overall, the results underscored the validity of the proposed deep learning framework because it estimated SBP, DBP, and HR simultaneously, a feature that may play an important role in clinically determining these physiological parameters among a more diverse groups of subjects with high accuracy. The proposed method performed better in measuring these parameters for patients with CVD complications than the existing methods, and it proved to have potential clinical applications. While deep learning methods have been found to work well in much of the literature, this study presented a deep learning framework that used single-channel PPG data to estimate DBP, SBP, and HR-with an NMAE of 0.0121, NRMSE of 0.0227, and R2_score of 0.97. Of all the loss functions used in the study, the MAE loss function worked best in our model. Preprocessing PPG data through a filter led to better DBP, SBP, and HR results. Moreover, as the results were obtained using a sample of patients with CVD complications, the proposed model may have good use in comprehensive medical monitoring. This model estimates BP values and the HR simultaneously, which makes it a more straightforward solution than the existing neural network models, which measure these parameters separately. The model also eliminates the need to perform feature selection and extraction separately (a common practice in conventional machine learning processes) because features are extracted during training. This helps to reduce the cost of data processing. Finally, in terms of the BHS standards, the model satisfies Grade A for DBP and DBP measurement.

Funding Statement: This study was supported in part by the Ministry of Science and Technology MOST108-2221-E-150-022-MY3 and National Taiwan Ocean University.

Conflicts of Interest: The authors declare that they have no conflicts of interest to report regarding the present study.

\section{References}

[1] C. El-Hajj and P. A. Kyriacou, "A review of machine learning techniques in photoplethysmography for the non-invasive cuff-less measurement of blood pressure," Biomedical Signal Processing and Control, vol. 58, pp. 1-13, 2020.

[2] L. Peter, N. Noury and M. Cernya, "A review of methods for non-invasive and continuous blood pressure monitoring: Pulse transit time method is promising?," Irbm, vol. 35, no. 5, pp. 271-282, 2014.

[3] A. Chandrasekhar, C. -S. Kim, M. Naji, K. Natarajan, J.-O. Hahn et al., "Smartphone-based blood pressure monitoring via the oscillometric finger-pressing method," Science Translational Medicine, vol. 10, no. 431, pp. eaap8674, 2018.

[4] K. -M. Tao, S. Sokha and H. B. Yuan, "Sphygmomanometer for invasive blood pressure monitoring in a medical mission," Anesthesiology, vol. 130, no. 2, pp. 312, 2019.

[5] D. Biswas, N. Simões-Capela, C. Van Hoof and N. Van Helleputte, "Heart rate estimation from wrist-worn photoplethysmography: A review," IEEE Sensors Journal, vol. 19, no. 16, pp. 6560-6570, 2019.

[6] A. B. Hertzman and C. R. Spielman, "Observations on the finger volume pulse recorded photo-electrically," American Journal of Physiology, vol. 119, pp. 334-335, 1937.

[7] C. Sideris, H. Kalantarian, E. Nemati and M. Sarrafzadeh, "Building continuous arterial blood pressure prediction models using recurrent networks," in 2016 IEEE Int. Conf. on Smart Computing (SMARTCOMP), St Louis, MO, USA, pp. 1-5, 2016.

[8] X. Li, S. Wu and L. Wang, "Blood pressure prediction via recurrent models with contextual layer," in Proc. of the 26th Int. Conf. on World Wide Web, Perth Australia, pp. 685-693, 2017.

[9] O. Schlesinger, N. Vigderhouse, D. Eytan and Y. Moshe, "Blood pressure estimation from PPG signals using convolutional neural networks and siamese network," in IEEE Int. Conf. on Acoustics, Speech and Signal Processing (ICASSP), Barcelona, Spain, pp. 1135-1139, 2020. 
[10] M. Panwar, A. Gautam, D. Biswas and A. Acharyya, "PP-Net: A deep learning framework for PPG-based blood pressure and heart rate estimation," IEEE Sensors Journal, vol. 20, no. 17, pp. 10000-10011, 2020.

[11] C. Fischer and T. Penzel, "Continuous non-invasive determination of nocturnal blood pressure variation using photoplethysmographic pulse wave signals: Comparison of pulse propagation time, pulse transit time and RR-interval,” Physiological Measurement, vol. 40, no. 1, pp. 014001, 2019.

[12] P. Li, M. Liu, X. Zhang, X. Hu, B. Pang et al., "Novel wavelet neural network algorithm for continuous and noninvasive dynamic estimation of blood pressure from photoplethysmography," Science China Information Sciences, vol. 59, pp. 2042405, 2016.

[13] A. Gaurav, M. Maheedhar, V. N. Tiwari and R. Narayanan "Cuff-less PPG based continuous blood pressure monitoring: a smartphone based approach," in Annual Int. Conf. of the IEEE Engineering in Medicine and Biology Society (EMBC), Orlando, FL, USA, pp. 607-610, 2016.

[14] M. Kachuee, M. M. Kiani, H. Mohammadzade and M. Shabany, "Cuffless blood pressure estimation algorithms for continuous health-care monitoring," in IEEE Transactions on Biomedical Engineering, vol. 64, no. 4, pp. 859-869, 2017.

[15] S. S. Mousavi, M. Firouzmand, M. Charmi, M. Hemmati, M. Moghadam et al., "Blood pressure estimation from appropriate and inappropriate PPG signals using a whole-based method," Biomed. Signal Process. Control, vol. 47, pp. 196-206, 2019.

[16] R. Miotto, F. Wang, S. Wang, X. Jiang and J. T. Dudley, "Deep learning for healthcare: Review, opportunities and challenges," Briefings in Bioinformatics, vol. 19, no. 6, pp. 1236-1246, 2018.

[17] D. Ravì, C. Wong, F. Deligianni, M. Berthelot, J. A. Perez et al., "Deep learning for health informatics," IEEE Journal of Biomedical and Health Informatics, vol. 21, no. 1, pp. 4-21, 2017.

[18] A. Jafari, A. Ganesan, C. S. K. Thalisetty, V. Sivasubramanian, T. Oates et al., "Sensornet: A scalable and Low-power deep convolutional neural network for multimodal data classification," IEEE Transactions on Circuits and Systems I: Regular Papers, vol. 66, no. 1, pp. 274-287, 2019.

[19] M. Panwar, S. R. Dyuthi, K. C. Prakash, D. Biswas, A. Acharyya et al., "CNN based approach for activity recognition using a wrist-worn accelerometer," in 2017 39th Annual Int. Conf. of the IEEE Engineering in Medicine and Biology Society (EMBC), Jeju, Korea (South), pp. 2438-2441, 2017.

[20] M. Panwar, D. Biswas, H. Bajaj, M. Jobges, R. Turk et al., "Rehab-net: Deep learning framework for Arm movement classification using wearable sensors for stroke rehabilitation," IEEE Transactions on Biomedical Engineering, vol. 66, no. 11, pp. 3026-3037, 2019.

[21] L. Everson, D. Biswas, M. Panwar, D. Rodopoulos, A. Acharyya et al., "Biometricnet: deep learning based biometric identification using wrist-worn PPG," in 2018 IEEE Int. Symposium on Circuits and Systems (ISCAS), Florence, Italy, pp. 1-5, 2018.

[22] C. J. Willmott, S. G. Ackleson, R. E. Davis, J. J. Feddema, K. M. Klink et al., "Statistics for the evaluation and comparison of models," Journal of Geophysical Research: Oceans, vol. 90, no. C5, pp. 8995-9005, 1985.

[23] J. Qi, J. Du, S. M. Siniscalchi, X. Ma and C. Lee, "Analyzing upper bounds on mean absolute errors for deep neural network-based vector-to-vector regression," IEEE Transactions on Signal Processing, vol. 68, pp. 3411-3422, 2020.

[24] E. Kyriakides, S. Suryanarayanan and G. T. Heydt, "State estimation in power engineering using the huber robust regression technique," IEEE Transactions on Power Systems, vol. 20, no. 2, pp. 1183-1184, 2005.

[25] N. Li, L. Wang, X. Li and Q. Zhu, "An effective deep learning neural network model for short-term load forecasting," Concurrency and Computation: Practice and Experience, vol. 32, no. 7, pp. e5595, 2020.

[26] E. Imani and M. White, "Improving regression performance with distributional losses," in 35th Int. Conf. on Machine Learning, 2018, Stockholm, Sweden, pp. 2157-2166, 2018.

[27] A. Pandey and D. Wang, "On adversarial training and loss functions for speech enhancement," in 2018 IEEE Int. Conf. on Acoustics, Speech and Signal Processing (ICASSP), Calgary, AB, Canada, pp. 5414 $5418,2018$.

[28] J. Qi, J. Du, S. M. Siniscalchi, X. Ma and C. -H. Lee, "On mean absolute error for deep neural network based vector-to-vector regression," IEEE Signal Processing Letters, vol. 27, pp. 1485-1489, 2020. 
[29] M. Kachuee, M. M. Kiani, H. Mohammadzade and M. Shabany, "Cuff-less high-accuracy calibrationfree blood pressure estimation using pulse transit time," in 2015 IEEE Int. Symp. on Circuits and Systems (ISCAS), Lisbon, Portugal, pp. 1006-1009, 2015.

[30] M. Saeed, M. Villarroe, A. T. Reisner, G. Clifford, L. W. Lehman et al., "Multiparameter intelligent monitoring in intensive care II: A public-access intensive care unit database," Critical Care Medicine, vol. 39, no. 5, pp. 952-60, 2011.

[31] G. Slapničar, N. Mlakar and M. Luštrek, "Blood pressure estimation from photoplethysmogram using a spectro-temporal deep neural network," Sensors, vol. 19, no. 15, pp. 3420, 2019.

[32] C. E. Hajj and P. A. Kyriacou, "Cuffless and continuous blood pressure estimation from PPG signals using recurrent neural networks," in 2020 42nd Annual Int. Conf. of the IEEE Engineering in Medicine \& Biology Society (EMBC), Montreal, QC, Canada, pp. 4269-4272, 2020.

[33] Z. Zhang, Z. Pi and B. Liu, "TROIKA: A general framework for heart rate monitoring using wristtype photoplethysmographic signals during intensive physical exercise," IEEE Transactions on Biomedical Engineering, vol. 62, no. 2, pp. 522-531, 2015.

[34] Z. Zhilin, "Photoplethysmography-based heart rate monitoring in physical activities via joint sparse spectrum reconstruction," IEEE Transactions on Biomedical Engineering, vol. 62, no. 8, pp. 1902-1910, 2015.

[35] E. Khan, F. A. Hossain, S. Z. Uddin, S. K. Alam and M. K. Hasan, "A robust heart rate monitoring scheme using photoplethysmographic signals corrupted by intense motion artifacts," IEEE Transactions on Biomedical Engineering, vol. 63, no. 3, pp. 550-562, 2016.

[36] A. Temko, "Accurate heart rate monitoring during physical exercises using PPG," IEEE Transactions on Biomedical Engineering, vol. 64, no. 9, pp. 2016-2024, 2017.

[37] L. Zhu, C. Kan, Y. Du and D. Du, "Heart rate monitoring during physical exercise from photoplethysmography using neural network," IEEE Sensors Letters, vol. 3, no. 1, pp. 1-4, 2019.

[38] K. R. Arunkumar and M. Bhaskar, "Heart rate estimation from photoplethysmography signal for wearable health monitoring devices," Biomedical Signal Processing and Control, vol. 50, pp. 1-9, 2019.

[39] D. Biswas, L. Everson, M. Liu, M. Panwar, B. E. Verhoef et al., "CorNET: Deep learning framework for PPG-based heart rate estimation and biometric identification in ambulant environment," IEEE Transactions on Biomedical Circuits and Systems, vol. 13, no. 2, pp. 282-291, 2019. 